\title{
Corrigendum: Usefulness and Potential Pitfalls of Long-Acting Growth Hormone Analogues
}

\section{OPEN ACCESS}

Edited by:

Ilonka Kreitschmann-Andermahr,

University of Duisburg-Essen,

Germany

Reviewed by:

Martin Bidlingmaier,

Ludwig Maximilian University of

Munich, Germany

Roberto Salvatori,

Johns Hopkins University,

United States

*Correspondence:

Kevin C. J. Yuen

kevin.yuen@dignityhealth.org

Specialty section: This article was submitted to Pituitary Endocrinology, a section of the journal Frontiers in Endocrinology

Received: 04 May 2021 Accepted: 24 May 2021 Published: 28 June 2021

Citation:

Yuen KCJ, Miller BS, Boguszewski CL and Hoffman AR (2021)

Corrigendum: Usefulness and

Potential Pitfalls of Long-Acting

Growth Hormone Analogues.

Front. Endocrinol. 12:705241.

doi: 10.3389/fendo.2021.705241
Kevin C. J. Yuen ${ }^{1 *}$, Bradley S. Miller ${ }^{2}$, Cesar L. Boguszewski ${ }^{3}$ and Andrew R. Hoffman ${ }^{4}$

1 Barrow Pituitary Center, Barrow Neurological Institute, Departments of Neuroendocrinology and Neurosurgery, University of Arizona College of Medicine and Creighton School of Medicine, Phoenix, AZ, United States, 2 Division of Pediatric Endocrinology, Department of Pediatrics, University of Minnesota, Minneapolis, MN, United States, ${ }^{3}$ SEMPR, Serviço de Endocrinologia e Metabologia, Departamento de Clínica Médica, Hospital de Clínicas da Universidade Federal do Paraná, Curitiba, Brazil, ${ }^{4}$ Department of Medicine, VA Palo Alto Health Care System and Stanford University School of Medicine, Palo Alto, CA, United States

Keywords: long-acting growth hormone, treatment adherence, growth hormone deficiency, growth hormone replacement, adults, children

\section{A Corrigendum on}

Usefulness and Potential Pitfalls of Long-Acting Growth Hormone Analogs by Yuen KCJ, Miller BS, Boguszewski CL, Hoffman AR. (2021). Front. Endocrinol. 12:637209. doi: 10.3389/fendo.2021.637209

\section{ERROR IN TABLE $1,5^{\text {TH }}$ COLUMN, $19^{\text {TH }}$ ROW}

In the original article, there was a mistake in Table $\mathbf{1}$ as published. In Table $\mathbf{1}, 5^{\text {th }}$ column, $19^{\text {th }}$ row, the company "Alteogen" under the "Current Status" column was incorrectly stated that the company was "bankrupt in 2009". This statement is incorrect as the company remains a currently viable bio-tech company globally.

The authors apologize for this inadvertent error with the statement and a modified Table $\mathbf{1}$ is provided below, where the statement "bankrupt in 2009" is now deleted. This new table does not change the scientific conclusions of the article in any way. The original article has been updated.

Copyright (C) 2021 Yuen, Miller, Boguszewski and Hoffman. This is an open-access article distributed under the terms of the Creative Commons Attribution License (CC BY). The use, distribution or reproduction in other forums is permitted, provided the original author(s) and the copyright owner(s) are credited and that the original publication in this journal is cited, in accordance with accepted academic practice. No use, distribution or reproduction is permitted which does not comply with these terms. 
TABLE 1 | Overview of the development history of LAGH analogues.

\begin{tabular}{|c|c|c|c|c|c|}
\hline Company & $\begin{array}{l}\text { LAGH } \\
\text { analog }\end{array}$ & $\begin{array}{l}\text { Modification to } \mathrm{GH} \\
\text { molecule }\end{array}$ & $\begin{array}{l}\text { Frequency of } \\
\text { administration }\end{array}$ & Current status & Research data \\
\hline \multicolumn{2}{|c|}{ Depot Formulation } & \multicolumn{4}{|l|}{ Depot Chemical } \\
\hline $\begin{array}{l}\text { Altus } \\
\text { Pharmaceuticals }\end{array}$ & ALTU-238 & $\begin{array}{l}\text { Long-extended release } \\
\text { formulation using protein } \\
\text { crystallization technology ( } 22 \\
\text { kDa) (39) }\end{array}$ & 7 days & Althea acquired assets in 2010 & No further studies planned \\
\hline $\begin{array}{l}\text { Critical } \\
\text { Pharmaceuticals }\end{array}$ & CP016 & $\begin{array}{l}\text { Supercritical carbon dioxide, } \\
\text { formed when } \mathrm{CO}_{2} \text { exceeds } \\
\text { its thermodynamic critical } \\
\text { point, used to create the } \\
\text { depot }(22 \mathrm{kDa})(39)\end{array}$ & 14 days & Company under liquidation & $\begin{array}{l}\text { Evidence of ongoing studies at other } \\
\text { corporations }\end{array}$ \\
\hline Genentech & $\begin{array}{l}\text { Nutropin } \\
\text { Depot }^{\circledR}\end{array}$ & $\begin{array}{l}\text { Encapsulated in } \\
\text { biocompatible, } \\
\text { biodegradable, polylactide- } \\
\text { coglycolide polymer } \\
\text { microsphere }(22 \mathrm{kDa})(40)\end{array}$ & 14 days & Removed from market (39) & \\
\hline $\begin{array}{l}\text { LG Life } \\
\text { Sciences, Ltd }\end{array}$ & $\begin{array}{l}\text { Eutropin } \\
\text { Plus }^{\text {TM }} \\
\text { (LB03002) }\end{array}$ & $\begin{array}{l}\text { Microparticles containing } \mathrm{GH} \\
\text { incorporated into sodium } \\
\text { hyaluronate and dispersed in } \\
\text { an oil base of medium-chain } \\
\text { triglycerides }(22 \mathrm{kDa})\end{array}$ & 7 days & $\begin{array}{l}\text { Marketed in Korea for childhood } \\
\text { GHD; approved in Europe but not } \\
\text { marketed in the EU }\end{array}$ & $\begin{array}{l}\text { Phase } 3 \text { trial in CGHD suggest non-inferiority } \\
\text { (41), safety data from a Korean registry } \\
\text { database in children with growth disorders } \\
\text { (42), Phase } 2 \text { trial in children with ISS } \\
\text { demonstrated non-inferiority and well- } \\
\text { tolerated (43) }\end{array}$ \\
\hline \multicolumn{2}{|c|}{ PEGylated Formulations } & \multicolumn{4}{|c|}{ PEGylation prolongs in vivo mean residence time of $\mathrm{GH}$, through slowing absorption and protection from proteolysis } \\
\hline Ambrx & ARX201 & $\begin{array}{l}\text { 30-kDa PEG added to } \\
\text { unnatural amino acid } \\
\text { incorporated into GH (52 } \\
\text { kDa) }\end{array}$ & 7 days & $\begin{array}{l}\text { No longer being developed (39) due } \\
\text { to PEGylated-containing vacuoles in } \\
\text { the epithelial cells of the choroid } \\
\text { plexus in monkeys (44) }\end{array}$ & \\
\hline $\begin{array}{l}\text { Bolder } \\
\text { BioTechnology }\end{array}$ & BBT-031 & $\begin{array}{l}\text { Site-specific PEGylated GH } \\
\text { analog (not available) }\end{array}$ & $\begin{array}{l}7 \text { days } \\
\text { (planned) }\end{array}$ & Preclinical studies (45) & \\
\hline $\begin{array}{l}\text { GeneScience } \\
\text { Pharmaceuticals } \\
\text { Co, Ltd }\end{array}$ & Jintrolong ${ }^{\circledR}$ & $\begin{array}{l}\text { 40-kDa PEG attached to } \mathrm{GH} \\
(62 \mathrm{kDa})\end{array}$ & 7 days $(13,16)$ & Marketed in China for CGHD & $\begin{array}{l}\text { Phase } 3 \text { studies show good IGF-I profile, } \\
\text { Phase } 4 \text { studies now ongoing }\end{array}$ \\
\hline Novo Nordisk & $\begin{array}{l}\text { NNC126- } \\
0083\end{array}$ & $\begin{array}{l}\text { 43-kDa PEG residue } \\
\text { attached to glutamine } 141 \\
(65 \mathrm{kDa})\end{array}$ & 7 days & $\begin{array}{l}\text { Unsatisfactory IGF-I profile peak and } \\
\text { duration (46) }\end{array}$ & No longer being developed as of 2011 \\
\hline Pfizer & PHA-794428 & $\begin{array}{l}\text { Branched } 40 \mathrm{kD} P E G \text { on } \mathrm{N} \text { - } \\
\text { terminus of } \mathrm{GH}(62 \mathrm{kDa})\end{array}$ & 7 days & $\begin{array}{l}\text { High rate of lipoatrophy at injection } \\
\text { site (47) }\end{array}$ & No longer being developed as of 2009 \\
\hline \multicolumn{2}{|c|}{ Pro-Drug formulation } & \multicolumn{4}{|c|}{ Mechanism of conversion to active drug } \\
\hline Ascendis & $\begin{array}{l}\text { TransCon } \\
\mathrm{GH}^{\circledR}(\mathrm{ACP}- \\
\text { 001) }\end{array}$ & $\begin{array}{l}\text { Unmodified rhGH transiently } \\
\text { bound to a PEG carrier } \\
\text { molecule via a self-cleaving } \\
\text { linker that is dependent upon } \\
\mathrm{pH} \text { and temperature ( } 22 \\
\mathrm{kDa})\end{array}$ & $\begin{array}{l}7 \text { days }(8,12, \\
14,18,48)\end{array}$ & $\begin{array}{l}\text { Phase } 2 \text { studies in CGHD and } \\
\text { AGHD showed comparable GH and } \\
\text { IGF-I profile to daily GH dosing } \\
\text { Phase } 3 \text { studies in CGHD showed } \\
\text { positive growth response (49) }\end{array}$ & $\begin{array}{l}\text { Completed Phase } 3 \text { study in CGHD and data } \\
\text { submitted to FDA and EMA } \\
\text { Phase } 3 \text { study in AGHD currently planned }\end{array}$ \\
\hline \multicolumn{2}{|c|}{$\begin{array}{l}\text { Non-covalent albumin } \\
\text { binding GH compound(s) }\end{array}$} & \multicolumn{4}{|l|}{ Albumin binding } \\
\hline $\begin{array}{l}\text { Novo Nordisk A } \\
\text { S }\end{array}$ & $\begin{array}{l}\text { Sogroya }^{\circledR} \\
\text { (NNC0195- } \\
\text { 0092) }\end{array}$ & $\begin{array}{l}\text { Single-point mutation in } \mathrm{GH} \text {, } \\
\text { with albumin binding moiety } \\
\text { attached (non-covalent } \\
\text { albumin-binding properties) } \\
(50,51)(23 \mathrm{kDa})\end{array}$ & 7 days (52) & $\begin{array}{l}\text { Phase } 2 \text { studies in CGHD showed } \\
\text { comparable IGF-I profile to daily GH } \\
\text { dosing (53) } \\
\text { Phase } 3 \text { studies in AGHD well } \\
\text { tolerated (54-56) } \\
\text { Approved by the FDA in August } \\
2020 \text { for use in AGHD but not } \\
\text { marketed yet }\end{array}$ & $\begin{array}{l}\text { Phase } 3 \text { studies in CGHD, Phase } 2 \text { studies in } \\
\text { SGA }\end{array}$ \\
\hline \multicolumn{2}{|c|}{ GH Fusion Proteins } & \multicolumn{4}{|l|}{ Protein fused with GH } \\
\hline $\begin{array}{l}\text { Ahngook } \\
\text { Pharmaceutical } \\
\text { Co, Ltd }\end{array}$ & AG-B1512 & $\begin{array}{l}\text { Recombinant GH genetically } \\
\text { fused to a polypeptide linker } \\
\text { and an anti-human serum } \\
\text { albumin Fab antibody ( } \sim 2 \\
\text { kDa) }\end{array}$ & $\begin{array}{l}14 \text { or } 28 \text { days } \\
(57)\end{array}$ & $\begin{array}{l}\text { Preclinical studies show IGF-I level } \\
\text { elevation sustained for } 20 \text { days }\end{array}$ & Ongoing research \\
\hline
\end{tabular}


TABLE 1 | Continued

\begin{tabular}{|c|c|c|c|c|c|}
\hline Company & $\begin{array}{l}\text { LAGH } \\
\text { analog }\end{array}$ & $\begin{array}{l}\text { Modification to GH } \\
\text { molecule }\end{array}$ & $\begin{array}{l}\text { Frequency of } \\
\text { administration }\end{array}$ & Current status & Research data \\
\hline Alteogen & ALT-P1 & $\begin{array}{l}\text { rhGH fused with NexP } \\
\text { recombinant a1-antitrypsin } \\
(\sim 74 \mathrm{kDa})(58)\end{array}$ & unknown & $\begin{array}{l}\text { Stopped Phase } 2 \text { study in CGHD } \\
\text { (59) }\end{array}$ & \\
\hline Asterion & $\begin{array}{l}\text { ProFuse }^{\mathrm{TM}} \\
\mathrm{GH}\end{array}$ & $\begin{array}{l}\text { GH binding protein }(\sim 82 \\
\mathrm{kDa})(60)\end{array}$ & $\begin{array}{l}1 \text { month } \\
\text { (planned) }\end{array}$ & $\begin{array}{l}\text { Preclinical studies to provide } \\
\text { intravascular stores of inactive GH }\end{array}$ & \\
\hline $\begin{array}{l}\text { Genexine and } \\
\text { Handok }\end{array}$ & $\mathrm{GX}-\mathrm{H} 9$ & $\begin{array}{l}\text { rhGH fused to hybrid non- } \\
\text { cytolytic immunoglobulin Fc } \\
\text { portions of lgD and lgG4 } \\
(100 \mathrm{kDa})(61)\end{array}$ & $7-14$ days (62) & $\begin{array}{l}\text { Phase } 2 \text { studies in AGHD completed } \\
(63) \\
\text { Phase } 2 \text { studies in CGHD showed } \\
\text { reassuring height changes }\end{array}$ & $\begin{array}{l}\text { Phase } 3 \text { studies in CGHD with twice-monthly } \\
\text { dosing ongoing }\end{array}$ \\
\hline $\begin{array}{l}\text { Hanmi } \\
\text { Pharmaceutical } \\
\text { Co }\end{array}$ & $\begin{array}{l}\text { LAPS rhGH } \\
\text { (HM10560A) }\end{array}$ & $\begin{array}{l}\text { Homodimeric aglycosylated } \\
\text { lgG4 Fc fragment ( } 51 \mathrm{kDa}) \\
\text { (64) }\end{array}$ & $7-14$ days $(64)$ & $\begin{array}{l}\text { Phase } 2 \text { in AGHD show good } \\
\text { tolerability }\end{array}$ & Phase 3 studies in AGHD (65) \\
\hline $\begin{array}{l}\text { JCR } \\
\text { Pharmaceuticals }\end{array}$ & $J R-142$ & $\begin{array}{l}\text { Engineered hGH fused at C- } \\
\text { terminus with modified } \\
\text { human serum albumin at } \mathrm{N} \text { - } \\
\text { terminus ( } \sim 8 \mathrm{kDa})(66)\end{array}$ & 7 days & Preclinical trials & Phase 1 study completed (67) \\
\hline Teva & $\begin{array}{l}\text { Albutropin } \\
\text { (TV-1106) }\end{array}$ & $\begin{array}{l}\text { Human serum albumin fused } \\
\text { to } N \text {-terminus of } \mathrm{GH}(88 \\
\mathrm{kDa} \text { ) }\end{array}$ & 7 days $(9,10)$ & $\begin{array}{l}\text { Studies in AGHD discontinued for } \\
\text { unknown reason; presumed } \\
\text { unfavorable benefit:risk profile }\end{array}$ & \\
\hline Versartis & $\begin{array}{l}\text { Somavaratan } \\
\text { (VRS-317) }\end{array}$ & $\begin{array}{l}\text { Fusion protein of rhGH and } \\
\text { the pharmacologically } \\
\text { inactive portion of long } \\
\text { chains of natural hydrophilic } \\
\text { amino acids (XTEN } \\
\text { technology) }\end{array}$ & $\begin{array}{l}7,14 \text { or } 28 \\
\text { days (22) }\end{array}$ & $\begin{array}{l}\text { No longer being developed as of } \\
2017 \text { as the Phase } 3 \text { study did not } \\
\text { meet its primary end-point for non- } \\
\text { inferiority comparison against daily } \\
\text { rhGH for height velocity in CGHD } \\
\text { (22) }\end{array}$ & \\
\hline
\end{tabular}

AGHD, adults with GH deficiency; CGHD, children with GH deficiency; EMA, European Medicines Agency; EU, European Union, FDA, Food and Drug Administration; kDa, kilodalton; ISS, idiopathic short stature; PEG, poly(ethylene glycol); rhGH, recombinant human GH; SGS, small for gestational age. Table is modified from Miller BS, et al. (70). 